\title{
Usefulness of the Twinkling Artifact in Identifying Implanted Mesh After Inguinal Hernia Repair
}

\author{
Gandikota Girish, MBBS, FRCS, FRCR, Elaine M. Caoili, MD, Amit Pandya, MD, Qian Dong, MD, \\ Michael G. Franz, MD, Yoav Morag, MD, Ellen J. Higgins, PA-C, Jonathan M. Rubin, MD, PhD, \\ David A. Jamadar, MBBS, FRCS, FRCR
}

Article includes CME test

Received February 2, 2011, from the Departments of Radiology (G.G., E.M.C., A.P., Q.D., Y.M., E.J.H., J.M.R, D.A.J.) and Surgery (M.G.F.), University of Michigan Hospitals, Ann Arbor, Michigan USA. Revision requested February 17, 2011. Revised manuscript accepted for publication March 16, 2011.

Wethank Brian Robertson, Tracy Boon, and Ilya Sedunova for their contribution to the understanding of the twinkling artifact produced by implanted inguinal mesh.

Address correspondence to Gandikota Girish, MBBS, FRCS, FRCR, Department of Radiology, University of Michigan Hospitals, 1500 EMedical Center Dr, Ann Arbor, MI 48109.

E-mail:ggirish@med.umich.edu
Objectives - Implanted mesh for inguinal hernia repair is often difficult to visualize with gray scale sonography and may present without the knowledge of the sonographer. We sought to evaluate the utility of the twinkling artifact produced by inguinal mesh to assist in mesh identification.

Methods - Two reviewers evaluated focused sonographic examinations of 44 inguinal regions, 24 of which had implanted inguinal mesh. The sonographic examinations consisted of static gray scale and color Doppler images with both linear and curvilinear array transducers. The presence of the twinkling artifact and visibility of the mesh were graded on a 4-point visibility scale.

Results - Inguinal mesh was not easily identified on gray scale imaging using either the curvilinear array $(P=.5)$ or linear array $(P=.5)$ transducer. The mesh was definitely seen in 3 of 24 inguinal regions using the linear array transducer and 2 of 24 inguinal regions using the curvilinear array transducer. In 79\% of inguinal regions with mesh, the twinkling artifact was produced with the curvilinear array transducer only. The artifact was not elicited when using the linear array transducer. With the use of the curvilinear array transducer and the presence of the twinkling artifact, there was a significant chance of correctly identifying the presence of mesh $(P<.005)$ in the entire study group.

Conclusions - Standard gray scale imaging alone is not reliable when identifying inguinal mesh. The twinkling artifact was present in $79 \%$ of inguinal regions with mesh when evaluated with a low-frequency curvilinear array transducer.

Key Words - inguinal hernia; mesh; twinkling artifact

$\mathrm{T}$ he surgical repair of inguinal hernias has evolved over the years. Today, most hernias are treated by placement of mesh $^{1-3}$ to repair an abdominal wall defect through which the herniated contents has passed. However, implanted mesh is a foreign body and is subject to a variety of complications, with recurrence remaining an unsolved problem and reoperation increasingly common. In the evaluation of these patients with implanted mesh, it is important to identify the mesh itself separate from the soft tissues that surround it to confirm a successful reconstruction, identify mesh failure, or better plan a salvage repair.

Twinkling is a sonographic artifact that may be seen behind a strongly reflective medium ${ }^{4,5}$ as a rapidly alternating red and blue signal. It can be observed just deep to the near-field interface of implanted mesh. 
Although gray scale sonography has been shown to identify nonmetallic foreign bodies, in our anecdotal experience, it has not been reliable in identifying mesh placed in the inguinal region. The purpose of this study was to evaluate the utility of the twinkling artifact in identifying patients with implanted mesh.

\section{Materials and Methods}

Institutional Review Board approval was obtained, and informed consent was waived for this retrospective study. From February 2009 to October 2009, 36 consecutive patients presenting either for evaluation for a recurrent hernia after mesh placement or with a de novo diagnosis of an inguinal hernia to be evaluated by sonography constituted the study group. Twenty-one of these patients had inguinal mesh ( 5 women and 16 men; age range, $25-71$ years; mean $\pm \mathrm{SD}, 53.9 \pm 11.1$ years), and 15 patients had no prior surgery ( 6 women and 9 men; age range, $18-68$ years; mean, $37.3 \pm 12.8$ years). Of the group with mesh, 3 patients had bilateral inguinal regions evaluated, and of the group without mesh, 5 patients had bilateral inguinal regions evaluated, resulting in 24 inguinal regions with inguinal mesh and a control group of 20 inguinal regions without inguinal mesh.

All studies were performed on an iU22 ultrasound machine (Phillips Healthcare, Bothell, WA). Static images that best represented the imaging findings were chosen for evaluation by a radiologist experienced in inguinal hernia sonography ( 5 years of experience). These images were obtained from both linear and curvilinear array transducers to best show mesh visibility with routine (gray scale) sonography and the twinkling artifact with color Doppler sonography. Comparable static images of inguinal regions without mesh implants were also collected. All images were then presented as unknowns to 2 experienced boardcertified radiologists (trained in body imaging), and any

Table 1. Inguinal Regions That Showed Visibility of Mesh on Gray Scale Imaging and Twinkling on Doppler Imaging in Patients With Implanted Mesh

\begin{tabular}{lrrrr}
\hline & \multicolumn{4}{c}{ Visibility Scale } \\
\cline { 2 - 5 } Imaging & $\mathbf{1}$ & $\mathbf{2}$ & $\mathbf{3}$ & $\mathbf{4}$ \\
\hline Linear gray scale & 3 & 10 & 6 & 5 \\
Curvilinear gray scale & 2 & 7 & 8 & 7 \\
Linear twinkling & 0 & 0 & 0 & 24 \\
Curvilinear twinkling & 19 & 0 & 0 & 5 \\
\hline
\end{tabular}

Values are numbers of cases that fit into the categories. Curvilinear indicates 5-2-MHz curvilinear array; linear, 12-MHz linear array; visibility scale: 1 , definitely seen; 2 , probably seen; 3 , not definitely seen; and 4 , not seen. disagreement between them was resolved by consensus. Before the evaluation, both radiologists were aware that about half of the images did not have mesh. These images were evaluated for the presence of mesh in the inguinal region (gray scale) and for the presence of the twinkling artifact. Visibility of the mesh and visibility of the twinkling artifact were graded on a 4-point scale, where 1 indicated definitely seen; 2 , probably seen; 3 , not definitely seen; and 4, not seen (Tables 1 and 2 ).

The inguinal region was evaluated with both a highfrequency $12-\mathrm{MHz}$ linear array transducer and a multifrequency $5-2-\mathrm{MHz}$ curvilinear array transducer. The sonographer performing the study was aware of the presence or absence of implanted mesh in the two groups of patients. The period between surgery and the examination ranged from 1 to 240 months (mean, 38.02 months), long enough for postoperative gas to be resorbed in all cases. In patients with implanted mesh, the twinkling artifact was evaluated using both transducers by scanning directly over the mesh. In those inguinal regions without mesh, interrogation of the inguinal region was in the axial plane, just cranial to the origin of the inferior epigastric artery for consistency of evaluation.

To show the twinkling artifact, a color Doppler region of interest was prescribed, and the pulse repetition frequency was increased to its highest level. This pulse repetition frequency for each transducer was set by the machine parameters and was operator independent. The color Doppler gain was increased to the point of "flare-out" and then decreased to just clear the area of interest, leaving the twinkling artifact, if present. Care was taken to differentiate the twinkling produced by adjacent bowel from the twinkling associated with implanted mesh. Bowel was identified by its relationship with the abdominal cavity, the presence of peristalsis, and the changing configuration with time.

Table 2. Inguinal Regions That Showed Visibility of Mesh on Gray Scale Imaging and Twinkling on Doppler Imaging in Control Patients Without Implanted Mesh

\begin{tabular}{lcccc}
\hline & \multicolumn{4}{c}{ Visibility Scale } \\
\cline { 2 - 5 } Imaging & $\mathbf{1}$ & $\mathbf{2}$ & $\mathbf{3}$ & $\mathbf{4}$ \\
\hline Linear gray scale & 0 & 1 & 4 & 15 \\
Curvilinear gray scale & 0 & 0 & 6 & 14 \\
Linear twinkling & 0 & 0 & 0 & 20 \\
Curvilinear twinkling & 0 & 0 & 0 & 20 \\
\hline
\end{tabular}

Values are numbers of cases that fit into the categories. Curvilinear indicates 5-2-MHz curvilinear array; linear, 12-MHz linear array; visibility scale: 1 , definitely seen; 2 , probably seen; 3 , not definitely seen; and 4, not seen. 
Information on the type of inguinal mesh used was only available in 14 of 24 inguinal regions. There were 2 cases in which 3D Max Mesh (Bard Davol, Inc, Warwick, RI) was used, 7 cases in which Parietex composite mesh (Covidien, Mansfield, MA) was used, 3 cases in which polypropylene mesh (C. R. Bard, Inc, Cranston, RI) was used, 1 case in which a PerFix plug (Bard Davol, Inc) was used, and 1 case in which Trelex Natural mesh (Maquet, Inc, Wayne, NJ) was used.

Evaluation of a sample of mesh (monofilament knitted polypropylene; C. R. Bard, Inc) by the same sonographic system in a water bath was also undertaken. The mesh sample was held suspended between two ceramic blocks, and it was allowed to protrude into the water bath. The bath was filled with tap water at room temperature, and the mesh was evaluated as detailed above.

Simple statistical analysis was performed with the Fisher exact test and Student $t$ test.

\section{Results}

On the basis of the Fisher exact test, there was no significant relationship between the presence of inguinal mesh and detection of mesh on gray scale sonography with either the linear array $(P=.5)$ or curvilinear array $(P=.5)$ transducer. The linear transducer showed marginally better numerical gray scale visibility than the curvilinear transducer (Table 1).

In $79 \%$ of the inguinal regions with mesh (19 of 24 ), a twinkling artifact was generated by the curvilinear array transducer $(P<.005)$. No twinkling artifact was generated by the linear array transducer in the presence of mesh. In the absence of mesh, the twinkling artifact was not generated by either transducer (Table 2 ).

Of the 24 inguinal regions with implanted mesh that were imaged with sonography for symptoms suspicious for hernia recurrence, 10 were found to have hernias at the margins of the mesh. These hernias did not interfere with mesh visualization.

When comparing the pulse repetition frequency of the linear array transducer in inguinal regions with mesh versus those without mesh, there was no significant difference ( $P=.37$, Student $t$ test). When comparing the pulse repetition frequencies of the curvilinear array transducer in inguinal regions with mesh versus those without mesh, there was no significant difference $(P>.06$, Student $t$ test $)$. However, when comparing the pulse repetition frequency between both the linear and curvilinear array transducers, there was a significant difference $(P=.003$, Student $t$ test). For inguinal regions with mesh: with the linear array trans- ducer, the mean pulse repetition frequency \pm SD was 8433 $\pm 1365.3 \mathrm{~Hz}$ (range, 3500-9000 Hz); with the curvilinear array transducer, the mean pulse repetition frequency was $7245.2 \pm 1295.8 \mathrm{~Hz}$ (range, 4800-9100 Hz). For inguinal regions without mesh: with the linear array transducer, the mean pulse repetition frequency was $8750 \pm 550.1 \mathrm{~Hz}$ (range, 7000-9000 Hz); with the curvilinear array transducer, the mean pulse repetition frequency was $8089.4 \pm$ $1547.4 \mathrm{~Hz}$ (range, 4950-11,700 Hz).

\section{Discussion}

Surgery for repair of inguinal hernias is a common procedure $^{6-8}$; most repairs in the United States involve the use of some synthetic mesh to buttress the defect or weakness in the abdominal wall. Although the widespread use of mesh repair of inguinal hernias has decreased recurrence rates, ${ }^{2}$ the mesh repair may fail and result in a recurrent hernia. ${ }^{9}$ Recurrent hernias are almost always found at the edge of implanted mesh. Mesh may become infected, migrate, ${ }^{10-14}$ or impinge on adjacent structures. ${ }^{15,16}$ Hematomas ${ }^{17-19}$ or seromas ${ }^{20}$ may develop, and the bowel may become adherent to the undersurface of the implanted mesh. ${ }^{21}$ The tacks holding the mesh in place may produce pain. ${ }^{22}$ Most of these complications of meshbased hernia repair have only further surgical solutions. Preoperative planning is important for surgical outcomes, and imaging should play a guiding role. As is the case in primary hernia repair, preoperative imaging may guide and improve the results of salvage reconstructions. The presence and location of implanted abdominal wall mesh can guide surgical diagnosis and therapy.

Sonography is a useful method for evaluating implanted mesh, 9,13,17 but frequently mesh may be difficult to visualize. Often the patient may not be aware of the details of the surgery performed, and knowledge of implanted mesh and clinical notes may not be available at the time of an examination. Mesh is usually seen as a curvilinear echogenic structure with posterior acoustic shadowing, ${ }^{13}$ but the narrow field of view of the transducer and the short depth of view may not allow the operator to perceive the shadowing easily. By widening the field of view and increasing the depth of view, ${ }^{23}$ the shadowing may be better appreciated. Occasionally, the implanted mesh may not be seen at all with sonography.

Twinkling is an artifact that is observed behind the near-field interface of a strongly reflective medium (Figure 1). The artifact itself is a rapidly alternating blue and red Doppler signal, which may appear to move and is seen just deep to the near-field interface of the object. ${ }^{4,5}$ The 


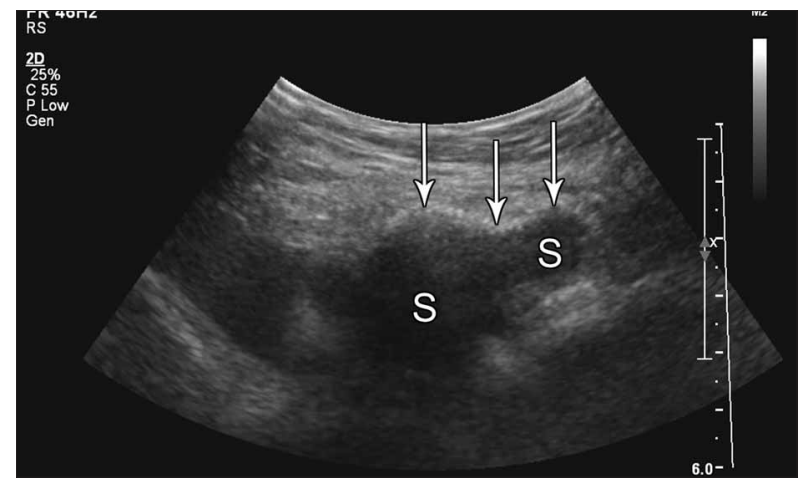

A

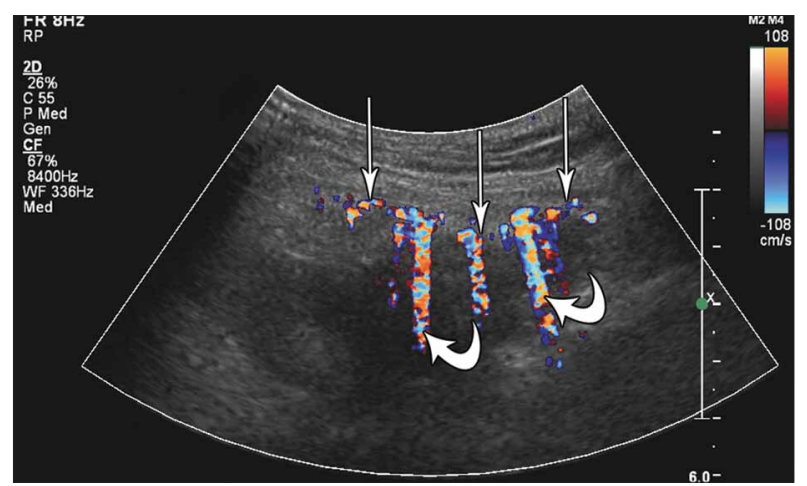

B

Figure 1. Images from a 47-year-old man with mesh repair of a right inguinal hernia and inguinal pain evaluated with a 5-2-MHz curvilinear array transducer. A, The echogenic wavy linear surface of the mesh implant (arrows) is easily seen with posterior acoustic shadowing (S). B, The twinkling artifact (curved arrows) is well seen extending from the mesh into the deeper tissue planes. The wavy contour of the mesh is still visible (straight arrows), although its linear echogenic surface is now not definitely seen.

cause is likely noise caused by phase (clock) jitter in the Doppler circuitry of the machine; a rough surface is thought to be secondary and broadens the spectrum. ${ }^{5} \mathrm{Al}-$ though this artifact may be found in different circumstances, ${ }^{24,25}$ the irregular surface of mesh also produces it, and this artifact may be useful in identifying mesh when it is not appreciated on routine gray scale sonography. The bowel wall may also show twinkling (Figure 2) and is a pitfall when evaluating mesh adjacent to the bowel, which usually occurs cranial to the implant when evaluating inguinal hernia mesh repairs.

In vitro, the twinkling artifact may be appreciated with both the low- and high-frequency transducers used in this study, although the artifact is more obvious with the lowerfrequency curvilinear array transducer (Figure 3). This finding of improved twinkling with lower-frequency trans- ducers and deep focusing has been described previously. ${ }^{26}$ In vivo, however, none of the 24 inguinal regions with inguinal mesh showed the twinkling artifact when the highfrequency linear array transducer was used, whereas most of the inguinal regions showed the artifact with the lowfrequency curvilinear array transducer (Table 1). We are uncertain of the reason for these results but suggest that incorporation into the soft tissues may in some way "smooth out" the irregularities of the mesh, making it less irregular and less prone to the artifact at higher frequencies. There is another potential contribution to twinkling that could explain these findings. Very strong signals can produce very unusual appearances in color Doppler images. This process is known as signal saturation, and saturated signals can produce very bizarre Doppler effects. ${ }^{25}$ Signal saturation happens because ultrasound Doppler/

Figure 2. Images from a 68-year-old man with right inguinal discomfort and implanted inguinal mesh evaluated with a 5-2-MHz curvilinear array transducer. A, The echogenic linear mesh (straight arrows) is somewhat difficult to see, and the acoustic shadowing (S) is more subtle than in Figure 1. The twinkling artifact is minimal and is immediately deep to the near-field interface of the mesh (curved arrow). B, Just cranial to the mesh implant, the adjacent bowel shows a marked twinkling artifact (curved arrows), a potential pitfall.

A

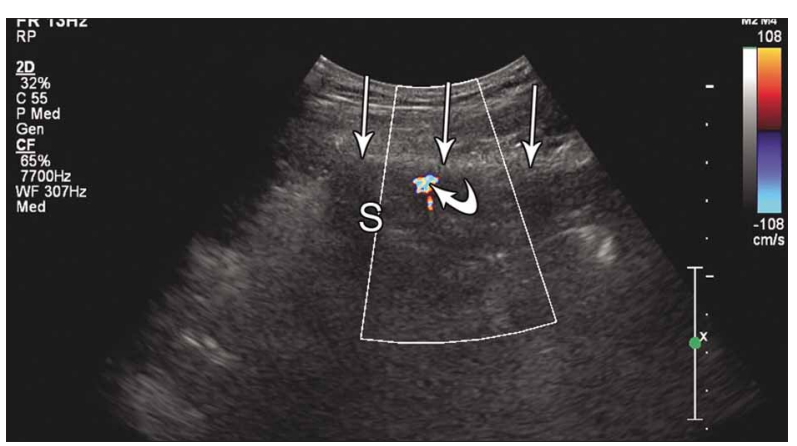

B

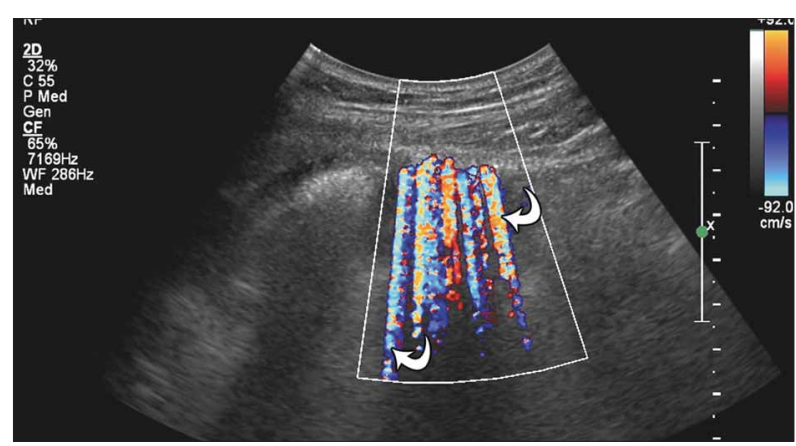


color Doppler processors expect very weak signals from blood, so if they get extremely strong signals from highly reflective surfaces such as bowel gas or stones, the Doppler signal-processing chain cannot handle these signals. Such signals may be represented in unusual ways, such as a highly fluctuating random color, just as with twinkling. Given that twinkling appears to be signal strength dependent, saturation could explain the differences seen here. Low frequencies are less attenuated than high frequencies, so backscattered signals will be much stronger using lowfrequency transducers. The exact contribution of signal saturation to twinkling and its relationship to surface roughness needs to be further investigated.

Nineteen of 24 inguinal regions with inguinal mesh showed a twinkling artifact with the low-frequency curvilinear array transducer. In 14 of 24 inguinal regions where information on the type of mesh implanted was available, we found that in all 5 inguinal regions with no twinkling, Parietex mesh (polyester mesh resorbable film) was implanted. However, 2 other inguinal hernias were repaired with the same mesh, which did show twinkling. The numbers were small, and it is uncertain what importance can be derived from them, but this question may be answered by a more detailed study.

In those cases in which a twinkling artifact was present, the readers were unequivocal in identifying the artifact $(P<.0002)$, even though the artifact was marked (Figure 1B) or minimal (Figure 2A). Although statistically significant, the readers were less certain in identifying the presence of mesh with gray scale imaging for both the linear and curvilinear array transducers. Using both the linear array high-frequency and the curvilinear array low-frequency

Figure 3. Mesh (monofilament knitted polypropylene; C. R. Bard, Inc, Cranston, RI) for repair of inguinal hernias scanned in a water bath with both $12-\mathrm{MHz}$ linear array and 5-2-MHz curvilinear array transducers. A, Linear array 12-MHz transducer showing the smooth linear echogenic mesh (arrows). Without circumferential support, the mesh assumes a mildly concave configuration. B, Twinkling occurs in the water bath with the linear array transducer (curved arrows) but is much less marked than with the curvilinear array transducer (see $\mathbf{D}$ ). The twinkling artifact partially obscures the echogenic (straight arrows) gray scale appearance of mesh. C. Curvilinear array 5-2-MHz transducer showing an appearance similar to that from the linear array transducer with gray scale sonography, the mesh appearing as a smooth linear echogenic surface (arrows). Without support, the mesh is concave. $\mathbf{D}$, The twinkling artifact $(T)$ with the curvilinear array transducer is marked and obscures the gray scale appearance of the mesh. The outline of the mesh is indicated by the arrows.

A

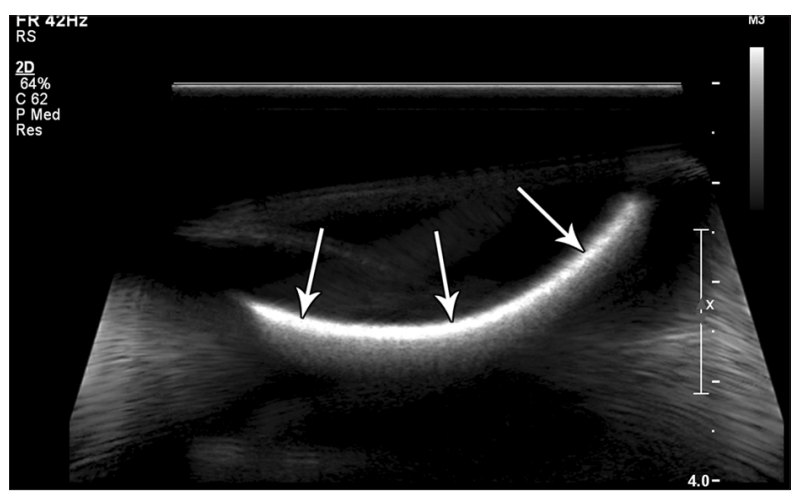

C

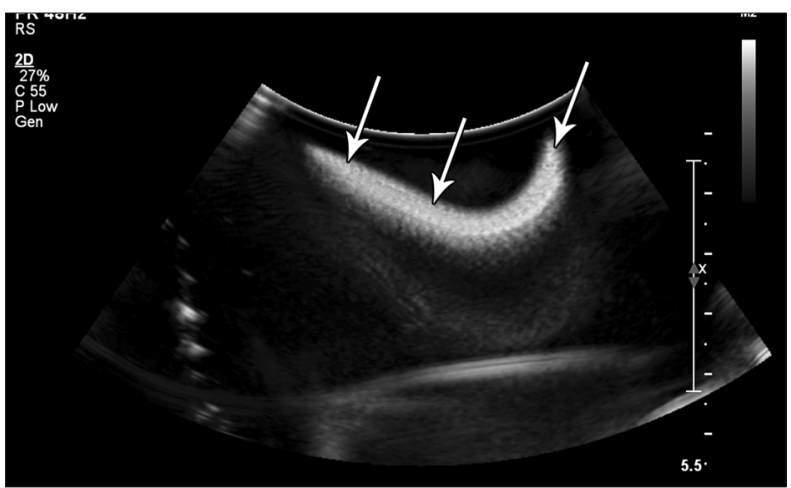

B



D

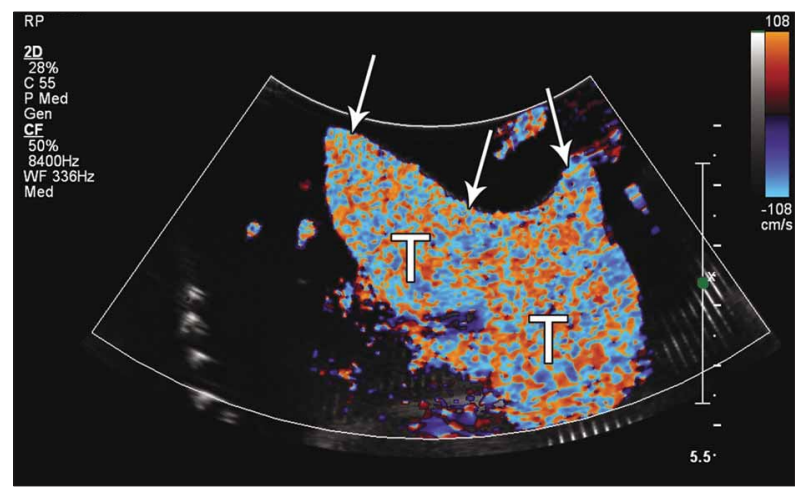


transducers, there were 2 inguinal regions (8\%) where mesh was not seen on gray scale imaging but a twinkling artifact was present and 3 additional inguinal regions $(12.5 \%)$ where the mesh was not definitely seen on gray scale imaging but a twinkling artifact was present. The twinkling artifact helped identify mesh in these $20.5 \%$ of inguinal regions.

In inguinal regions with absent mesh, there was 1 inguinal region where mesh was thought to have probably been seen on gray scale sonography. In the remainder of these inguinal regions with no implanted mesh, the readers correctly did not see the mesh in most of them, although in some it was thought not definitely seen (Table 2 ). These findings emphasize the difficulty in confidently identifying implanted inguinal mesh, or its absence, with gray scale sonography. Of the 5 inguinal regions with inguinal mesh and no twinkling artifact, 3 were either not seen or not definitely seen on gray scale sonography using the linear transducer, the curvilinear transducer, or both (Table 1). Although this group was a small subset of the inguinal regions evaluated, it was not insubstantial and reflects the ongoing difficulty in identifying inguinal mesh.

Limitations of this study included its retrospective nature and the use of static images to make a routine sonographic assessment in evaluating for the presence of mesh. Our experience is that the identification of implanted inguinal mesh by static images is more difficult than with real-time gray scale sonography, perhaps making assessment for mesh by readers more difficult. However, static images allowed consistency and facilitated the provision of blinded material for evaluation. The sonographer performing the study was aware of the presence or absence of implanted mesh before the examinations, which may have resulted in some bias, although the twinkling artifact when present was definitely visualized, likely minimizing this bias. The broad range of pulse repetition frequencies reported was probably related to the depth of the color box, which varied from patient to patient, a limitation of a retrospective study. Compounding was not turned off for the gray scale images, which would make the perception of acoustic shadowing more difficult to appreciate. However, given the size of the implanted mesh used in these repairs, this factor might not necessarily have affected the perceptions and interpretations of the readers. A high-frequency linear array transducer was compared with a low-frequency curvilinear array transducer, but this process was thought to reflect common clinical practice and answered our clinical question. The small numbers of the study were a final limitation.
In conclusion, the use of a low-frequency curvilinear transducer and the twinkling artifact may help identify the presence of implanted mesh after inguinal hernia repair in some cases in which the implant is difficult to appreciate with gray scale sonography.

\section{References}

1. Stickel M, Rentsch M, Clevert DA, et al. Laparoscopic mesh repair of incisional hernia: an alternative to the conventional open repair? Hernia 2007; 11:217-222.

2. van Veen RN, Wijsmuller AR, Vrijland WW, Hop WC, Lange JF, Jeekel J. Long-term follow-up of a randomized clinical trial of nonmesh versus mesh repair of primary inguinal hernia. BrJ Surg 2007; 94:506-510.

3. Luijendijk RW, Hop WCJ, van den Tol MP, et al. A comparison of suture repair with mesh repair for incisional hernia. N Engl J Med 2000; 343:392-398

4. Rahmouni A, Bargoin R, Herment A, Bargoin N, Vasile N. Color Doppler twinkling artifact in hyperechoic regions. Radiology 1996; 199:269-271.

5. Kamaya A, Tuthill T, Rubin JM. Twinkling artifact on color Doppler sonography: dependence on machine parameters and underlying cause. AJR Am J Roentgenol 2003; 180:215-222.

6. Rutkow IM. Surgical operations in the United States: then (1983) and now (1994). Arch Surg 1997; 132:983-990.

7. Rutkow IM. Demographic and socioeconomic aspects of hernia repair in the United States in 2003. Surg Clin North Am 2003; 83:1045-1051.

8. Ruhl CE, Everhart JE. Risk factors for inguinal hernia among adults in the US population. Am J Epidemiol 2007; 165:1154-1161.

9. ParraJA, Revuelta S, Gallego T, Bueno J, Berrio JI, Farinas MC. Prosthetic mesh used for inguinal and ventral hernia repair: normal appearance and complications in ultrasound and CT. Br J Radiol 2004; 77:261-265.

10. Dieter RAJr. Mesh plug migration into the scrotum: a new complication of hernia repair. Int Surg 1999; 84:57-59.

11. Chowbey PK, Bagchi N, Goel A, et al. Mesh migration into the bladder after TEP repair: a rare case report. Surg Laparosc Endosc Percutan Tech 2006; 16:52-53

12. Celik A, Kutun S, Kockar C, Mengi N, Ulucanlar H, Cetin A. Colonoscopic removal of inguinal hernia mesh: report of a case and literature review. J Laparoendosc Adv Surg Tech A 2005; 15:408-410.

13. Crespi G, Giannetta E, Mariani F, Floris F, Pretolesi F, Marino P. Imaging of early postoperative complications after polypropylene mesh repair of inguinal hernia. Radiol Med 2004; 108:107-115.

14. Murphy JW, Misra DC, Silverglide B. Sigmoid colonic fistula secondary to Perfix-plug, left inguinal hernia repair. Hernia 2006; 10:436-438.

15. Jamadar DA, Jacobson JA, Girish G, et al. Abdominal wall hernia mesh repair: sonography of mesh and common complications. J Ultrasound Med 2008; 27:907-917.

16. Stout CL, Foret A, Christie DB, Mullis E. Small bowel volvulus caused by migrating mesh plug. Am Surg 2007; 73:796-797. 
17. Furtschegger A, Sandbichler P, Judmaier W, Gstir H, Steiner E, Egender G. Sonography in the postoperative evaluation of laparoscopic inguinal hernia repair.J Ultrasound Med 1995; 14:679-684.

18. Wicks JD, Silver TM, Bree RL. Gray scale features of hematomas: an ultrasonic spectrum. AJR Am J Roentgenol 1978; 131:977-980.

19. Rankin RN, Hutton L, Grace DM. Postoperative abdominal wall hematomas have a distinctive appearance on ultrasonography. Can J Surg $1985 ; 28: 84-85$.

20. Susmallian S, Gewurtz G, Ezri T, Charuzi I. Seroma after laparoscopic repair of hernia with PTFE patch: is it really a complication? Hernia 2001; 5:139-141.

21. Arnaud JP, Hennekinne-Mucci S, Pessaux P, Tuech JJ, Aube C. Ultrasound detection of visceral adhesions after intraperitoneal ventral hernia treatment: a comparative study of protected versus unprotected meshes. Hernia 2003; 7:85-88.

22. Topart P,Vandenbroucke F, Lozach P. Tisseel versus tack staples as mesh fixation in totally extraperitoneal laparoscopic repair of groin hernias: a retrospective analysis. Surg Endosc 2005; 19:724-727.

23. Jamadar DA, Franz MG. Inguinal region hernias. In: Jacobson JA (ed). Ultrasound Clinics: Musculoskeletal Ultrasound. Philadelphia, PA: WB Saunders Co; 2007:711-725.

24. Reuben CM, Jeun B, Juluru K. Sonographic twinkling artifact in a renal graft with prolene mesh. J Ultrasound Med 2010; 29:1011-1013.

25. Kim HC, Yang DM, Jin W, Ryu JK, Shin HC. Color Doppler twinkling artifacts in various conditions during abdominal and pelvic surgery. J Ultrasound Med 2010; 29:621-632.

26. Tchelepi H, Ralls PW. Color comet-tail artifact: clinical applications. AJR Am J Roentgenol 2009; 192:11-18. 